\title{
THE USES OF MELATONIN
}

Dimah Sweis

ack of sleep in children is a very difficult stress factor, especially in the case of children with disabilities. This can have severe detrimental effects on the family, particularly when already -experiencing the stresses related to caring for a disabled child. Several approaches are available comprising both pharmacological and behavioural techniques. The exogenous use of melatonin, a hormone that is naturally found in the brain, has recently been gaining increasing attention in the treatment of many sleep disorders. ${ }^{1}$ The following review has been prepared exploring the current uses of melatonin in the paediatric population. A literature search in Medline and the Cochrane Library was conducted, utilising keywords such as "melatonin", "children", "paediatrics", "uses", "sleep disorders", "EEG", and "epilepsy", and produced 61 references that were useful in compiling this review.

\section{MELATONIN: A NATURAL HORMONE}

Melatonin is a natural hormone that is synthesised in the pineal gland in the brain. Described as the "Dark Force", ${ }^{2}$ melatonin is heavily dependent on the circadian rhythm generated by the light/dark cycle. ${ }^{3}$ This endogenous clock in the human brain induces the secretion of melatonin at night, reaching maximum plasma concentrations at 3-4 am. When the retinas in the eyes detect light, melatonin synthesis is inhibited, leading to secretion only when the subject is in darkness. In humans, melatonin is first detected in babies of 3-6 months. Production increases to a maximum at $1-5$ years, and begins to decrease around the beginning of puberty. ${ }^{4}$

Melatonin was first isolated in the 1960s by Lerner ${ }^{5}$ who documented its hypnotic effects. Interest in this substance gradually began to increase, reaching a peak in the 1990s when melatonin was described as a "miracle drug" used to treat everything from jetlag to cancer as well as possessing an anti-aging effect. ${ }^{6}$ Although many of these claims were disputed, ${ }^{7}$ its hypnotic effect when used for the treatment of sleep disorders has gained increasing credibility. ${ }^{8}$

Since then, the use of melatonin in children has become well documented, particularly in anecdotal reports and small studies. ${ }^{8-10}$ Particular uses emerged, specifically in treatment of sleep disorders ${ }^{811-13}$ and epilepsy. ${ }^{4}{ }^{14}$ Melatonin appeared to be especially targeted for blind children ${ }^{10}{ }^{15}$ and those with neurodevelopmental disabilities. ${ }^{12}{ }^{16-18}$

Despite the extensive use and small studies surrounding melatonin, it remains an unlicensed medicine in the UK. There is little official information available regarding the safety and efficacy of melatonin use in adults or children. Although many small studies and case reports had noted some impact from melatonin, there were others that found it to be mostly ineffective. ${ }^{9}$ There has been no clear evidence of adverse events mentioned in any of the studies, although this does not rule out the possibility of rare or long term effects; particular concern was voiced regarding delayed puberty in pre-pubertal children. ${ }^{10}$ In addition, there is no universal agreement on the dose of melatonin that should be used, with studies reporting doses ranging from $0.3-500 \mathrm{mg}$ of fast release melatonin. ${ }^{19}{ }^{20}$ Many of these obscurities could be resolved by conducting randomised controlled trials for melatonin in children with neurodevelopmental disabilities and a wide range of sleep problems. Unfortunately, it has been shown that obtaining sponsorship for a randomised controlled trial (RCT) on melatonin is very difficult ${ }^{21}$ considering the nature of this product, its over the counter status in some countries, and the age group being tested.

\section{USES OF MELATONIN \\ Sleep disorders}

Bedtime struggling and night awakenings have been known to affect about $20 \%$ of the general paediatric population. ${ }^{12}$ These disturbances could be a result of many causes such as pain, gastrooesophageal reflux, excessive noise, illness, seizures, and emotional and other endogenous or environmental factors ${ }^{13}$ Many times, sleep disorders are caused by circadian disturbances, whereby the sleep-wake rhythm is unsynchronised, leading to sporadic sleeping patterns. Often such disorders are associated with neurodevelopmental disabilities, epilepsy, and visual disturbances. ${ }^{22}$ When attempting to treat sleeplessness in children, particularly with neurodevelopmental disorders, the first step is to obtain an accurate sleep history documenting the child's 
typical sleep schedule. This would be essential to obtain a precise diagnosis of the sleep disorder before commencement of specific treatment. ${ }^{23}$ Many times, a clear diagnosis of the sleep disorder may be difficult to obtain, particularly in children with many neurodevelopmental disabilities.

Regardless of the cause of these sleep problems, the primary approach to enhance the child's sleep must always be through non-pharmacological means. Several strategies have been suggested, including sleep scheduling, bedtime routine plus gradual distancing of parents, bedtime fading with or without response cost, and chronotherapy. ${ }^{1}$

Several studies have been conducted investigating the use of melatonin in children with sleep disorders. ${ }^{10} 1322$ These studies found that administration of melatonin improved sleep initiation and continuity throughout the night. Children fell asleep quickly and there was a decrease in the frequency of nocturnal awakenings. ${ }^{24}$ In some children, their sleep difficulties disappeared after the first dose, while others began to have better sleeping patterns within days and sometimes weeks. ${ }^{13}$ A retrospective study also concluded that melatonin use on these children is safe, effective, and well tolerated. It also found melatonin to be beneficial in children with attention deficit hyperactivity disorder (ADHD), mood and anxiety disturbances, depression, as well as healthy children with insomnia. ${ }^{22}$ The dose of melatonin in these studies ranged from $0.3-6 \mathrm{mg}$ of fast release melatonin 30 60 minutes before bedtime. Once the sleep pattern improved, some patients were able to withdraw treatment successfully over a period of several months. ${ }^{13}$

\section{Visual impairment}

It is believed that endogenous melatonin secretion is regulated by the amount of light on the retina of the eyes. Therefore, children who are visually impaired may not perceive the light-dark alterations which synchronise the chronological and physiological day, leading to sleep-wake disturbances. ${ }^{19}$ Hung et $a l^{16}$ noticed that children with visual impairment, irrespective of type or cause, appeared to show the best response, whereas no obvious consistent pattern of response was seen in children who had normal visual function. Other studies that included blind children also reported significant improvement in sleep patterns of children with visual impairment. ${ }^{1013}{ }^{19}$ It was suggested that melatonin be given 20-30 minutes before bedtime, with one study using fast release doses of $0.5-4 \mathrm{mg}$ while another administered $2.5-10 \mathrm{mg}$.

\section{Neurological disorders}

Up to $80 \%$ of children with neurodevelopmental disabilities are known to suffer from sleep disturbances that are resistant to sedatives and hypnotics. ${ }^{12}$ Because of the severity of their illness, it is sometimes very difficult to enforce sleep habits on these children. Consequent to findings that exogenous melatonin is beneficial in chronic sleep-wake cycle disorder, several researchers attempted to investigate the use of melatonin in children with neurological disorders. Such studies involved children experiencing multiple disabilities including mental handicaps, visual impairment, and epilepsy.

These studies showed remarkable improvement in the children, ${ }^{11}$ documenting quick and long lasting benefit in $80 \%$ of the participants. ${ }^{25}$ Hung et $a l^{16}$ noted that melatonin appeared to induce an "all or nothing" response, where the children either benefited greatly from the treatment, or experienced no notable improvement. Only one study ${ }^{9}$ found melatonin to be ineffective in patients with fragmented sleep. This may be due to the low doses administered in this study. Camfield et $a l^{9}$ only used 0.5-1 mg melatonin, whereas the remaining studies increased doses up to a maximum of 9 mg. ${ }^{11}$

There were no reported adverse reactions in these studies, unlike other hypnotics that lead to a "hangover" effect. ${ }^{8}$ Palm et $a l^{10}$ included some patients who remained on melatonin for six years, with no reported long term effects. Only one study reported an increase in seizure activity after treatment in four of the six children who participated in spite of improved sleep patterns. ${ }^{26}$

\section{Epilepsy}

Melatonin was first reported to improve significantly clinical electroencephalogram (EEG) patterns in untreatable epileptic patients with different aetiologies by Anton Tay et al in $1974 .^{27}$ There are several explanations for the effect of melatonin on epilepsy. It is known to have a neuroprotective role, due to its ability to inhibit glutamate receptors and potentiate GABAbenzodiazepine receptors. ${ }^{19}$ Melatonin is also known as an antioxidant, scavenging highly toxic free radicals that are generated during seizures. ${ }^{14}$ Finally, it is known that epilepsy is exacerbated by lack of sleep, therefore improved sleep patterns would lead to fewer seizures. ${ }^{24}$

Many investigations have been carried out examining the effect of melatonin on children with epilepsy. These studies have shown very contrasting results, whereby some have found significant improvement, while others reported remarkable deterioration. Several studies have found contrasting results within the same investigation, reporting random cases of worsening and improvement. ${ }^{11}{ }^{16}$ One study ${ }^{26}$ described worrying results that seizure frequency increased with melatonin administration, in spite of improved sleep patterns. Discontinuation of treatment led to the return of seizure activity to baseline, and re-challenge with melatonin caused a recurrence of seizures. It must be noted that this study only included six children, which is not a representative sample, and there have been no other studies that reported similar results.

Alternatively, several studies have reported significant decreases in seizure frequency confirming the anticonvulsant action of melatonin, ${ }^{4}{ }^{19}{ }^{24}$ particularly for myoclonus seizures and nocturnal seizures. ${ }^{20}{ }^{28}$ Melatonin has also been investigated as adjunctive treatment to valproate ${ }^{14}$ and phenobarbital. ${ }^{20}$ Such studies showed favourable effects of melatonin, leading to lower anticonvulsant doses, thereby reducing the side effects.

The above results suggest the need for further investigation regarding the effect of melatonin on epilepsy.

\section{Sleep EEG}

EEG readings are routinely taken to monitor and detect epileptiform abnormalities in epileptic patients. This entails obtaining recordings while the patient is in a light sleep state. If a patient is not expected to sleep spontaneously, sleep can be prompted by administering a mild soporific agent, or by sleep deprivation. Either method may have a direct effect on the EEG reading. ${ }^{29}$ In addition, if a child requires sleep deprivation, this may be extremely stressful for the child and the family. ${ }^{29}$

Melatonin was introduced as a potentially suitable alternative to induce mild sleep in patients. When tried in adults and compared to administration of secobarbital, melatonin 
was found to be equally effective, and did not display any EEG manifestations that are typical of barbiturate effects. ${ }^{30}$ Subsequently, a similar study was conducted in children. As before, melatonin induced EEGs proved to be a suitable alternative and did not affect the EEG readings. ${ }^{29}$ It was found to be more acceptable to the family of the child than

\section{GUIDELINES FOR ADMINISTRATION}

Melatonin is currently available in either fast release or controlled release preparations. Fast release preparations have been reported to be more effective for treatment of delayed sleep onset whereas controlled release melatonin is more useful for sleep maintenance. Most studies with melatonin have only used the fast release formulation; however Jan et $a l^{24}$ conducted a clinical trial specifically to assess the efficacy of controlled release melatonin on sleepwake cycle disorders in children. Controlled release formulations were found to have a slightly later sleep onset and were most useful in improving sleep fragmentation and early morning awakening. Alternatively, fast release formulations were more effective for children with delayed sleep onset, but appeared to have little or no effect after 5-6 hours. Jan et al ${ }^{24}$ found that the most effective average dose of controlled release melatonin was $5.7 \mathrm{mg}$, which was slightly lower than the $7 \mathrm{mg}$ dose determined for fast release melatonin.

\section{Sleep disorders}

There were many variations in the dosing regimens used by researchers when investigating the use of melatonin in children. Literature shows that children require a higher dose of melatonin than do adults. ${ }^{24}$ This is probably because children metabolise this hormone faster before puberty. There is also evidence that there is a maximum saturation dose of the melatonin receptors, after which no increased effect can be observed. This was seen when testing large doses (10-240 mg); it was found that melatonin effect was not related to the dose. ${ }^{31}$

Most studies used doses ranging between $0.5-10 \mathrm{mg}$ in children, depending on age. ${ }^{13}{ }^{16}{ }^{19}$ Initial recommended doses were 1-3 mg for infants and toddlers, and 2.5-5 mg for older children. Depending on the response in the first few days, dose can be increased gradually to a maximum of $10 \mathrm{mg} .{ }^{13}$ If the child was only experiencing delayed sleep onset, then the fast release formulation would provide sufficient results. However, children who also experience sleep fragmentation and nocturnal awakenings may benefit from a combination of fast release and controlled release melatonin given at bedtime. ${ }^{24}$

Melatonin is given 20-30 minutes before bedtime, and children fall asleep within one hour. ${ }^{22}{ }^{24}$ It is recommended that melatonin be given on an empty stomach or with minimal fluids, since the absorption of oral melatonin may be delayed when taken with large meals. It can also interfere with other medication such as anticonvulsants, so they should be given one or more hours before melatonin administration. ${ }^{13}$ After acceptable sleep patterns are attained for six months, it may be possible to attempt a withdrawal of melatonin ${ }^{16}$ by gradually decreasing the dose over several weeks. However, sometimes sleep disorders may recur. ${ }^{13} 19$

\section{Epilepsy}

Several studies on children with epilepsy were conducted using varying doses of melatonin. One recommendation was

that fast release melatonin is administered one hour before bedtime using the following doses ${ }^{12}$ :

- $6 \mathrm{mg}$ for children younger than 9 years or weighing $<30 \mathrm{~kg}$

- $9 \mathrm{mg}$ for children older than 9 years or weighing $>30 \mathrm{~kg}$

In another study, children were started on $5 \mathrm{mg}$ and if there was no improvement after 3-5 days the dose was slowly increased to a maximum of $10 \mathrm{mg}{ }^{4}$

One particular study attempted to initiate melatonin in an untreatable child with severe myoclonic epilepsy as an adjunctive to anticonvulsant therapy. ${ }^{20}$ In this particular study, the child was given an initial dose of $500 \mathrm{mg}$ which triggered a convulsive seizure lasting three hours. After one week, another attempt was made to start melatonin with a dose of $50 \mathrm{mg}$, resulting in a notable decrease in the number of seizures. After much experimentation, the optimal dosing regimen for this child was $120 \mathrm{mg}$ /day (20 mg at 9 am and $100 \mathrm{mg}$ at $9 \mathrm{pm}$ ). When the melatonin dose was reduced, seizures were more likely to occur, and the melatonin concentrations were to be maintained high throughout the entire day.

\section{Sleep EEG}

Melatonin administered for sleep EEG is only required on the day of the EEG reading. The fast release formulation was given $(2-10 \mathrm{mg})$ depending on the age of the child, just before the EEG recording. Children tended to fall asleep in an average of 33 minutes after melatonin was administered. It was also found that EEGs carried out in the afternoon were more effective; more children fell asleep and sleep onset was earlier. ${ }^{29}$

\section{ADVERSE REACTIONS}

Most of the studies conducted on melatonin have found no significant adverse reactions to this hormone. ${ }^{13} 14162232$ Jan et $a l^{24}$ have been studying the effects of melatonin in children since 1991, including some who were taking this medicine for 5-7 years, and have reported no adverse reactions related to growth or puberty of these children. In addition, a recent clinical trial found no toxicological effects from melatonin. ${ }^{33}$ However, it is recommended that pregnant women should not receive exogenous melatonin until much more is known about its effect on the fetus. ${ }^{13}$

\section{CONCLUSIONS}

Melatonin has been shown to have promising effects in multi-disabled children experiencing sleep disorders. There is also increasing evidence of its efficacy in treating sleep disorders related to specific illnesses ${ }^{19}$ such as Angelman syndrome, $^{34}$ Rett syndrome, ${ }^{35}$ and tuberous sclerosis. ${ }^{36}$ Although further studies are recommended, it appears to be safe and well tolerated.

Melatonin treatment should be conducted with prudence; it should not be used as a blanket treatment for every sleep disturbance, although it seems to offer a suitable alternative to other sedatives and hypnotics. It can also be used to assist in teaching children better sleeping habits and in specific circumstances such as illness, trips, or changes in routine such as vacations. Children who have benefited from this treatment became much calmer, happier, playful, and affectionate. ${ }^{13}$ For many parents who have suffered with their child through sleep disorders, they have described melatonin as a "miracle drug". 


\section{REFERENCES}

1 Lancioni GE, O'Reilly MF, Basili G. Review of strategies for treating sleep problems in persons with severe or profound mental retardation or multiple handicaps. Am J Mental Retard 1999;104:170-86.

2 Bergstrom WH, Hakanson DO. Melatonin: the Dark Force. In: Barness LA, eds. Advances in pediatrics. Mosby Inc, 1998:91-107.

3 Geoffriau M, Brun J, Chazot G, et al. The physiology and pharmacology of melatonin in humans. Hormone Research 1998;49:136-141.

4 Fauteck JD, Schmidt H, Lerchl A, et al. Melatonin in epilepsy: first results of replacement therapy and first clinical results. Biological Signals and Receptors 1999;8:105-10

5 Lerner AB. My 60 years in pigmentation - Aaron B. Lerner. Pigment Cell Research 1999;12:131-44.

6 Pierpaoli W, Regelson W, Colman C. The melatonin miracle: nature's age reversing, disease-fighting, sex-enhancing hormone. New York: Simon \& Schuster Inc, 1996.

7 Bonn D. Melatonin's multifarious marvels: miracle or myth? Lancet 1996;347:184.

8 Jan JE, Espezel H, Appleton RE. The treatment of sleep disorders with melatonin. Dev Med Child Neurol 1994;36:97-107.

9 Camfield P, Gordon K, Dooley J, et al. Melatonin appears ineffective in children with intellectual deficits and fragmented sleep: six " $\mathrm{N}$ of 1 " trials. J Child Neurol 1996;11:341-3.

10 Palm L, Blennow G, Wetterberg L. Long term melatonin treatment in blind children and young adults with circadian sleep-wake disturbances. Dev Med Child Neurol 1997;39:319-25.

11 Coppola G, lervolino G, Mastrosimone M, et al. Melatonin in wake-sleep disorders in children, adolescents and young adults with mental retardation with or without epilepsy: a double-blind, cross-over, placebo-controlled trial. Brain Dev 2004;26:373-6

12 Jan JE, Odonnell ME. Use of melatonin in the treatment of paediatric sleep disorders. J Pineal Res 1996;21:193-9.

13 Jan JE, Freeman RD, Fast DK. Melatonin treatment of sleep-wake cycle disorders in children and adolescents. Dev Med Child Neurol 1999;41:491-500

14 Gupta M, Aneja S, Kohli K. Add-on melatonin improves quality of life in epileptic children on valproate monotherapy: a randomized, double-blind, placebo-controlled trial. Epilepsy \& Behavior 2004:5:316-21.

15 Cavallo A, Good WV, Douglas Ris M, et al. Dose response to melatonin treatment for disordered sleep rhythm in a blind child. Sleep Medicine 2002;3:159-61.

16 Hung JC, Appleton RE, Nunn AJ, et al. The use of melatonin in the treatment of sleep disturbances in children with neurological or behavioural disorders. The Journal of Pediatric Pharmacy Practice 1998;3:250-6.

17 Owens JA, Maxim R, Nobile C, et al. Parental and self-report of sleep in children with attention- deficit/hyperactivity disorder. Arch Pediatr Adolesc Med 2000;154:549-55.
18 Willey C, Phillips B. Is melatonin likely to help children with neurodevelopmental disability and chronic severe sleep problems? Arch Dis Child 2002;87:260.

19 Gordon N. The therapeutics of melatonin: a paediatric perspective. Brain Dev 2000;22:213-17.

20 MolinaCarballo A, MunozHoyos A, Reiter RJ, et al. Utility of high doses of melatonin as adjunctive anticonvulsant therapy in a child with severe myoclonic epilepsy: two years' experience. J Pineal Res 1997;23:97-105.

21 Sweis D. Exploration on the use of unlicensed and off-label medicines in children (PhD thesis). University of Bradford, 2004.

22 Ivanenko A, Crabtree VM, Tauman R, et al. Melatonin in children and adolescents with insomnia: a retrospective study. Clin Pediatr 2003;42:51-8.

23 Hoban TF. Sleeplessness in children with neurodevelopmental disorders epidemiology and management. CNS Drugs 2000;14:11-22.

24 Jan JE, Hamilton D, Seward N, et al. Clinical trials of controlled-release melatonin in children with sleep-wake cycle disorders. J Pineal Res 2000;29:34-9.

25 Jan MM. Melatonin for the treatment of handicapped children with severe sleep disorders. Pediatr Neurol 2000;23:229-32.

26 Sheldon SH. Pro-convulsant effects of oral melatonin in neurologically disabled children. Lancet 1998;351:1254.

27 Anton-Tay F. Melatonin: effects on brain function. Adv Biochem Psychopharmacol 1974;11:315-24

28 Jan JE, Connolly MBC, Hamilton D, et al. Melatonin treatment of non-epileptic myoclonus in children. Dev Med Child Neurol 1999:41:255-9.

29 Wassmer E, Carter PFB, Quinn E, et al. Melatonin is useful for recording sleep EEGs: a prospective audit of outcome. Dev Med Child Neurol $2001 ; 43: 735-8$

30 Milstein V, Small JG, Spencer DW. Melatonin for sleep EEG. Clin Electroencephalogr 1998;29:49-53.

31 Zhdanova IV, Wurtman RJ, Lynch HJ, et al. Sleep-inducing effects of low doses of melatonin ingested in the evening. Clin Pharmacol Ther 1995; 57:552-8

32 Ross C, Davies P, Whitehouse W. Melatonin treatment for sleep disorders in children with neurodevelopmental disorders: an observational study. Dev Med Child Neurol 2002;44:339-44.

33 Seabra ML, Bignotto M, Pinto LR Jr, et al. Randomized, double-blind clinical trial, controlled with placebo, of the toxicology of chronic melatonin treatment. J Pineal Res 2000;29:193-200.

34 Zhdanova IV, Wurtman RJ, Wagstaff J. Effects of a low dose of melatonin on sleep in children with Angelman syndrome. J Pediatr Endocrinol Metab 1999:12:57-67.

35 McArthur AJ, Budden SS. Sleep dysfunction in Rett syndrome: a trial of exogenous melatonin treatment. Dev Med Child Neurol 1998;40:186-92.

36 O'Callaghan FJK, Clarke AA, Hancock E, et al. Use of melatonin to treat sleep disorders in tuberous sclerosis. Dev Med Child Neurol $1999 ; 41: 123-6$

11 th European Forum on Quality Improvement in Health Care

26-28 April 2006, Prague, Czech Republic

For further information please go to: www.quality.bmjpg.com

Book early to benefit from a discounted delegate rate 